\title{
Commute-Time Convolution Kernels for Graph Clustering
}

\author{
Normawati A. Rahman and Edwin R. Hancock \\ Department of Computer Science, University of York, \\ Heslington, York, YO10 5DD, UK
}

\begin{abstract}
Commute time has proved to be a powerful attribute for clustering and characterising graph structure, and which is easily computed from the Laplacian spectrum. Moreover, commute time is robust to deletions of random edges and noisy edge weights. In this paper, we explore the idea of using convolution kernel to compare the distributions of commute time over pairs of graphs. We commence by computing the commute time distance in graphs. We then use a Gaussian convolution kernel to compare distributions. We use kernel kmeans for clustering and use kernel PCA for illustration using the COIL object recognition database.
\end{abstract}

Keywords: commute times, laplacian, graph kernel, convolution kernel.

\section{Introduction}

There has recently been a concerted effort in the literature to extend the kernel paradigm from pattern-vectors to relational structures such as graphs, trees and strings. In particular, various types of graph kernel have been suggested, with specific goals in mind [5891113/4151824. Generally speaking, there are two sources of information that can be exploited in the construction of a graph kernel. Firstly, there is information concerning the structure of the graph. This can be encapsulated in a number of ways. However, one of the most powerful is to use information concerning the distribution of path length or the frequency of different cycle lengths. The second source of information is conveyed by the labels or attributes on the nodes or edges of a graph. Finally, there are correspondences between the nodes of the graphs being compared. There have been many examples of the use of kernels in conjunction with graphs for instance Smola et al [2], develop path-length kernels and use them from comparing molecular structures. Gartner et al [11, use kernels for mining graphs from large databases. Bunke and Riesen [3] have shown how kernel methods can be used to transform pattern analysis problems using graphs into equivalent statistical pattern analysis tasks.

These three sources of information are obtained at different cost and play different roles. For instance, reliable node correspondences are both difficult and costly to locate. If correspondences are to hand, then the attributes can provide powerful discriminating information. In fact, the use of correspondences can be viewed as implicitly vectorising the available attribute information for the graphs 
under study. Relational structure, on the other hand is something that is intrinsic to graphs, and distinguishes them from data in the form of vectors or strings.

The aim in this paper is to explore the extent to which structural information alone can be used to construct a kernel, and the kernel used for the purposes of graph clustering. We require a structural characterisation which is both economically computed and robust to minor perturbations in graph structure due to noise. The characterisation also needs to be fine enough so as to distinguish reasonably subtle changes in structure. Path lengths provide one candidate which have been extensively explored in the graph kernels literature. Examples include the path-length kernel [123] and the diffusion map [16. However, commute time [19. provides an interesting alternative that captures the features of these two alternatives in a robust way. The commute time is the expected number of steps for a random walk to travel from one node of a graph to another, and then return again. The quantity is averaged over all possible paths. As a result it is relatively robust to edge deletion. It can also be shown to average the diffusion map over all possible diffusion lengths. Moreover, it is a metric and can be simply computed from the Laplacian spectrum in a time that is cubic in the number of nodes in the graph.

To avoid the need for explicit correspondences, we make use of the convolution kernel. Our idea is to compute the commute time between all pairs of nodes in a graph using the Laplacian spectrum, yielding a commute time matrix. We then compare the elements of the commute time matrices using the convolution kernel. This avoids explicit element by element correspondences, which would normally require the estimation of a permutation matrix between the nodes of the graph. Instead the convolution matrix weights against commute times that differ in magnitude. Viewed in this way the convolution kernel has features reminiscent of the computation of Hausdorff distance between graphs [12].

\section{Commute Time on Graph}

We commence by briefly reviewing the relationship between the Laplacian spectrum and the commute time. Consider the weighted graph $G=(V, E, \Omega)$ where $V$ is the set of nodes, $E \subseteq V \times V$ is the set of edges and $\Omega: E \rightarrow[0,1]$ is the set of weights associated with the edges. The adjacency matrix of the graph has elements

$$
A_{u v}= \begin{cases}1 & \text { if }(u, v) \in E \\ 0 & \text { otherwise }\end{cases}
$$

Let $T=\operatorname{diag}\left(d_{u} ; u \in V\right)$ be the diagonal weighted degree matrix with elements $\sum_{v=1}^{n} A(u, v)$, where $n=|V|$. The Laplacian matrix is given by $L=T-A$. The normalized weighted Laplacian matrix is defined to be $\mathrm{L}=T^{-1 / 2}(T-A) T^{-1 / 2}$, and has elements

$$
\mathrm{L}_{u v}=\left\{\begin{array}{cl}
1 & \text { if } u=v \\
-\frac{w_{u, v}}{\sqrt{d_{u} d_{v}}} & \text { if } u \neq v \text { and }(u, v) \in E \\
0 & \text { otherwise, }
\end{array}\right.
$$


The spectral decomposition of the normalized Laplacian is $\mathrm{L}=\Phi \Lambda \Phi^{T}$, where $\Lambda=\operatorname{diag}\left(\lambda_{1}, \lambda_{2}, \ldots . \lambda_{n}\right)$ is the diagonal matrix with the eigenvalues as elements satisfying the ordering $0=\lambda_{1} \leq \lambda_{2} \ldots \leq \lambda_{|V|}$ and $\Phi=\left(\phi_{1}\left|\phi_{2}\right| \ldots \mid \phi_{|V|}\right)$ is the matrix with the correspondingly ordered eigenvectors as columns.

The Green's function $\Gamma$ is the pseudo inverse of the normalized Laplacian matrix, which is computed by discarding the eigenvector associated with the zero eigenvalue, i.e.

$$
\Gamma(u, v)=\sum_{i=2}^{|V|} \frac{1}{\lambda_{i}} \phi_{i}(u) \phi_{i}(v)
$$

The expected number of steps taken by a random walk to reach node $v$, commencing from node $u$ is defined as the hitting time $O(u, v)$, and the commute time $C T(u, v)$ is the expected time for a random walk to travel from node $u$ to reach node $v$ and then return. Thus, $C T(u, v)=O(u, v)+O(v, u)$, and it has been shown in [4, that it can be computed using the Green's function $\Gamma$ by,

$$
C T(u, v)=\operatorname{vol}\left(T^{-1 / 2}(\Gamma(u, u)+\Gamma(v, v)-2 \Gamma(u, v)) T^{-1 / 2}\right)
$$

where $v o l$ is the volume of the graph. It has been proven in 4], that by substituting the spectral expression for the Green's function into the definition of the commute time, it is straightforward to show that in terms of the eigenvalues and eigenvectors of the normalized Laplacian [19],

$$
C T(u, v)=v o l \sum_{i=2}^{|V|} \frac{1}{\lambda_{i}}\left(\frac{\phi_{1}(u)}{\sqrt{d_{u}}}-\frac{\phi_{i}(v)}{\sqrt{d_{v}}}\right)^{2}
$$

Thus, the only operation required to compute the commute time matrix is the extraction of the eigenvectors and eigenvalues of the normalized Laplacian $L$. The commute time is a metric on the graph. In the next section, we'll show how we use the commute time matrix to compare the distribution of this metric over the graphs.

\section{Convolution Kernels on Graph}

Our objective in this paper is to use the graph kernel to compare the distribution of the commute time over the graphs and hence gauge graph similarity. One way to do this is by constructing a convolution graph kernel between the two graphs being considered.

Consider two graphs $G_{1}=\left(V_{1}, E_{1}\right)$ and $G_{2}=\left(V_{2}, E_{2}\right)$ with commute time matrices $C T_{1}$ and $C T_{2}$, representing the distributions of commute time within each graph. We wish to work without locating explicit correspondences between nodes of the two graphs. Hence, we make use of the convolution kernel to compare the commute-time matrices.

Convolution kernels were introduced by Haussler [10]. Consider two patternvectors $\boldsymbol{x}=x_{1}, \ldots x_{D}$ and $\boldsymbol{y}=y_{1}, \ldots y_{D}$. Suppose that for each corresponding pairs of elements $\left(x_{d}, y_{d}\right)$ where $1 \leq d \leq D$, we have a kernel $K_{d}$ that can be 
used to measure their similarity, i.e. $K_{d}\left(x_{d}, y_{d}\right)$. The similarity $K(x, y)$ between the two pattern-vectors $\boldsymbol{x}$ and $\boldsymbol{y}$ is given by the following generalized convolution

$$
K(x, y)=\int_{\boldsymbol{x}} \int_{\boldsymbol{y}} \prod_{d=1}^{D} K_{d}\left(x_{d} y_{d}\right) d \boldsymbol{y} d \boldsymbol{x}
$$

We can use any positive definite kernel with this definition. Here we use the Gaussian kernel to compare the commute time matrices for the graphs on an element by element basis. As a result the convolution kernel is

$$
K\left(G_{1}, G_{2}\right)=\frac{1}{\left|V_{1}\right|} \frac{1}{\left|V_{2}\right|} \sum_{(a, b) \in V_{1} \times V_{1}} \sum_{(\alpha, \beta) \in V_{2} \times V_{2}} \exp ^{-\frac{\left|C T_{1}(a, b)-C T_{2}(\alpha, \beta)\right|^{2}}{2 \sigma^{2}}}
$$

We have applied kernel PCA 21] to the convolution kernel for visualisation. Kernel PCA is the extension of PCA to a kernel feature space. Kernel kmeans has been well used for clustering. Here, we would like to cluster our kernel matrix based on kernel kmeans algorithm. Kernel kmeans [20] is a generalization of the standards kmeans algorithm where data points from input space are mapped into higher dimensional feature space through a nonlinear transformation $\phi$ and then kmeans is applied in the feature space. Clustering a test set of objects using the kernel kmeans approach involves partitioning the dataset into $M$ disjoint clusters.

\section{Experiment}

In this section, we provide some experimental evaluation of our proposed method. In our experiments, we use two datasets. The first of these is the COIL [17. database of 72 views of each of a number of objects (see Figure 1). The second is a database containing 72 views of four toys (example images are shown in Figure 21). The datasets contain multiple images of objects as the camera pans around the object. Corner features for the COIL data and SIFT features for the toys, are extracted from the images and Delaunay graphs representing the arrangement of feature points are constructed.

We commence the experiment by computing the commute time distance for the graphs based on equation (3). With the value of the commute time at hand, we construct the kernel matrix based on equation (5). Then the data is embedded in a low dimensional space using kernel PCA and the kernel k-means algorithm used for clustering.

We apply kernel PCA to the output of the convolution kernel using the algorithm described in 22 and obtain an embedding of the graph data. Figure 3 shows the results obtained. Here the different coloured points refer to different objects. We also compare our method with the shortest path kernel on a graph and the modified Hausdorff distance. The path kernels used were constructed by computing the product of kernel on edges encountered along the walk. Here, we only take the edge weights into consideration in order to compute the path kernel. This is because we work with unlabeled graphs in the implementation of 

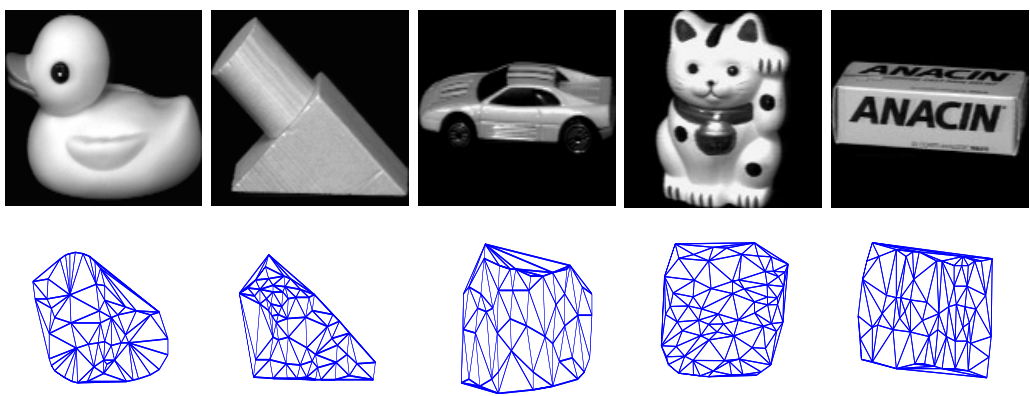

Fig. 1. Coil images that are used for the experiment and the corresponding delaunay graphs
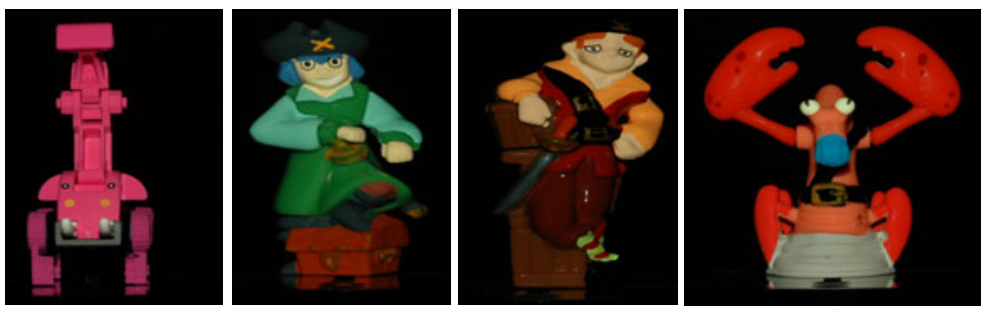

Fig. 2. Examples images from the toys database

our kernel based on commute time matrix, and we wish to maintain comparability. The results for the path-length kernel are shown in Figure 4. The results obtained using the modified Hausdorff are shown in Figure 5. Here we compute distances between the commute time matrices using the modified Hausdorff distance reported in [7] and then embed the graphs into a low dimensional pattern space using multidimensional scaling 6. Here we compare the results for both unweighted and weighted graphs.

From visual inspection of the results, there is some suggestion that the convolution kernel gives better results than the alternative two methods. Specifically, the different objects are separated into clearer clusters. To be more quantitative, we use the kernel K-means to perform clustering. Here we consider the first 5 objects from the COIL database. We use Rand index to validate the cluster. Rand index measures the consistency of a given clustering, therefore higher values indicate better clustering. We regard all pairs of objects $\left(o_{i}, o_{j}\right)$ with $o_{i} \neq o_{j}$ for computing the Rand index. We denote pairs of object $\left(o_{i}, o_{j}\right)$ that belong to same class and to same cluster with $N_{1}$, whereas $N_{2}$ denotes the number of pairs that neither belong to the same class nor to the same cluster. The number of pairs belonging to the same class but not to the same cluster is denoted as $N_{3}$, while $N_{4}$ represents the number of pairs belonging to different class but same cluster. The Rand index is thus defined as Rand index $=\left(N_{1}+N_{2}\right) /\left(N_{1}+N_{2}+N_{3}+N_{4}\right)$. 

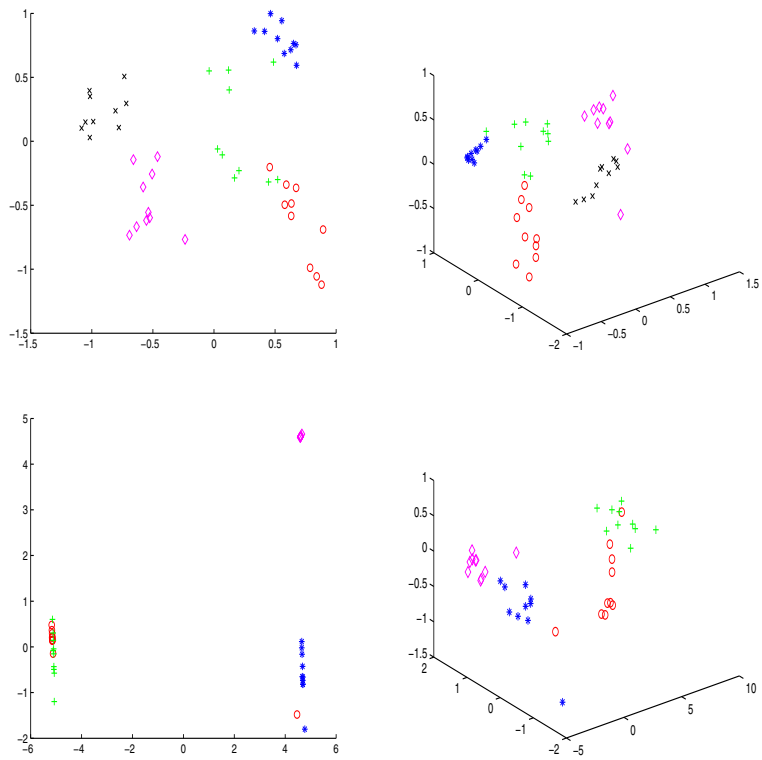

Fig. 3. Kernel PCA embedding of Gaussian convolution kernel using commute time as features.Top: Coil database. Bottom: Toys Database.
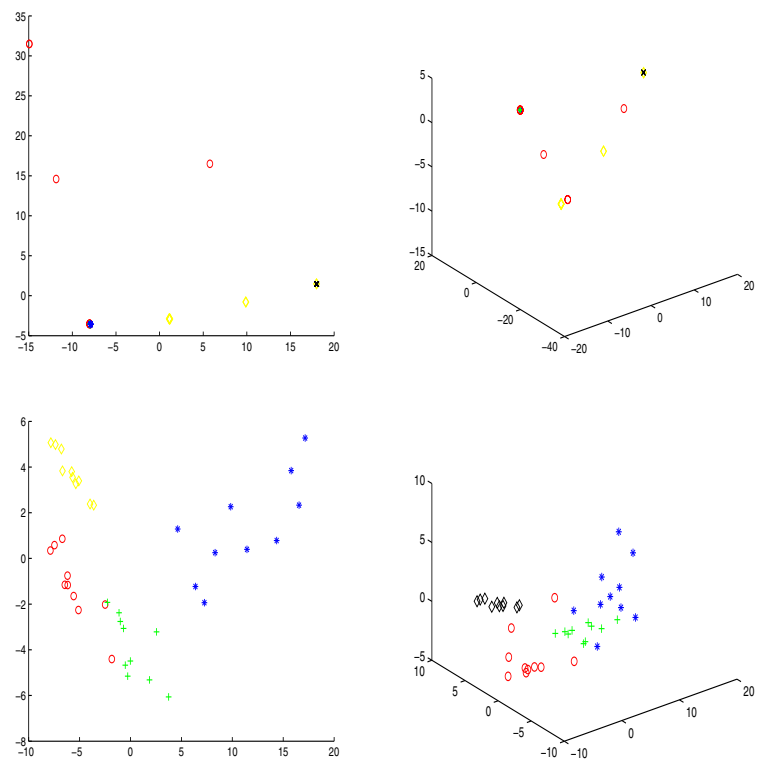

Fig. 4. Kernel PCA embedding of shortest path kernel. Top: Coil database. Bottom: Toys Database. 

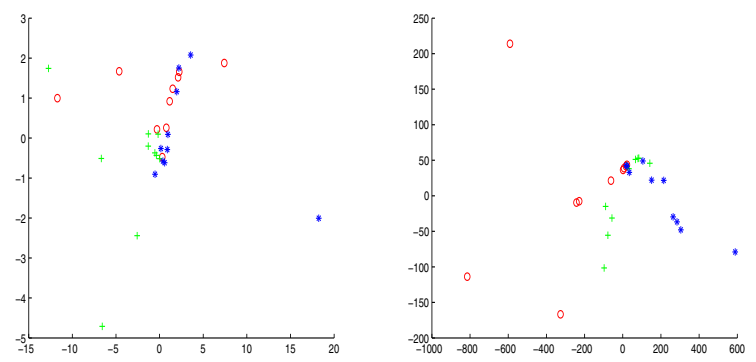

Fig. 5. Modified Hausdorff distance of Commute Time using MDS embedding. Left:Unweighted graph. Right: Weighted graph.

Table 1. Rand index for different value of $\sigma$

\begin{tabular}{|l|c|c|c|}
\hline \multicolumn{4}{|c|}{ Rand index } \\
\hline Data & $\sigma=0.1$ & $\sigma=1$ & $\sigma=10$ \\
\hline COIL data using commute time kernel & 0.7954 & 0.7286 & 0.6587 \\
\hline COIL data using path length kernel & 0.7202 & 0.6714 & 0.6714 \\
\hline toys data using commute time kernel & 0.7670 & 0.6330 & 0.6330 \\
\hline toys data using path length kernel & 0.6770 & 0.6670 & 0.6670 \\
\hline
\end{tabular}

Table 1 shows the Rand index for the COIL and toys dataset computed using commute time kernel and path length kernel with different value for $\sigma$.

From table 1. we can see that the performance of commute time kernel is comparable to shortest path kernel if not better as it gives higher values when $\sigma$ is close to 0.1 for both COIL and toys datasets.

\section{Conclusion}

We have shown how the distribution of the commute time between pairs of vertices in the graph can be used to compute a measure of similarity between graphs using the convolution kernel. We have illustrated the effectiveness of the similarities on graph clustering and classification experiments. The commute time between vertices can be used as a more robust measure of vertex affinity than the path length distance as it is more robust to errors in edge weight structure.

\section{References}

1. Kriegel, H.P., Borgwardt, K.M.: Shortest path kernels on graphs. In: Proceedings of the Fifth IEEE International Conference on Data Mining (ICDM 2005), pp. 74-81 (2005)

2. Ong, C.S., Schönauer, S., Vishwanathan, S.V.N., Smola, A.J., Kriegel, H.-P., Borgwardt, K.M.: Protein function prediction via graph kernels. In: In Proceedings of Intelligent Systems in Molecular Biology (ISMB), Detroit, USA (2005) 
3. Bunke, K., Riesen, H.: A family of novel graph kernels for structural pattern recognition. LNCS, pp. 20-31. Springer, Heidelberg (2007)

4. Yau, S.T., Chung, F.: Discrete green's function. Journal of Combinatorial Theory Series A 91(1-2), 191-214 (2004)

5. Duffy, N., Collins, M.: Convolution kernels for natural language. Advances in Neural Information Processing Systems 14 (2002)

6. Cox, M.A.A., Cox, T.F.: Multidimensional Scaling. Chapman and Hall, Boca Raton (2001)

7. Jain, A., Dubuisson, M.: A modified haursdoff distance for object matching. In: Proc. 12th Int. Conf. Pattern Recognition, pp. 566-568 (1994)

8. Flach, P., Wrobel, S., Gartner, T.: On graph kernels: Hardness results and efficient alternatives. In: Scholkopf, B., Warmuth, M. (eds.) Sixteen Annual Conference on Computational Learning Theory and Seventh Kernel Workshop, COLT (2003)

9. Gartner, T.: Exponential and geometric kernels for graphs. In: NIP 2002 Workshop on Unreal Data, Volume Principles of Modelling Nonvectorial Data (2002)

10. Haussler, D.: Convolution kernels on discrete structure. Technical report, UCSCCRL-99-10, UC Sansta Cruz (1999)

11. Gartner, T., Wrobel-S. Hovarth, T.: Cyclic pattern kernels for predictive graph mining. In: Proceedings of International Conference on Knowloedge Discovery and Data Mining (KDD), pp. 158-167 (2004)

12. Hancock, E.R., Huet, B.: Relational object recognition from large structural libraries. Pattern Recognition 35(9), 1895-1915 (2002)

13. Tsuda, K., Inokuchi-A. Kashima, H.: Marginalized kernels between labelled graphs. In: In Proceedings of the 20th International Conference on Machine Learning (ICML), Washington, DC, United States (2003)

14. Saunders, C., Shawe-Taylor, -J., Christianini, N., Watkins, C., Lodhi, H.: Text classification using string kernels. Journal of Machine Learning Research 2, 419-444 (2002)

15. Ueda, N., Akutsu, -T., -Perret, J.-L., Vert, J.-P., Mahe, P.: Extensions of marginalized graph kernels. In: ICML (2004)

16. Lafon, S., coifman, -R.R., -Kevrekidis, I.G., Nadler, B.: Diffusion maps, spectral clustering and eigenfunctions of fokker-planck operators. In: Advances in Neural Information Processing Systems, vol. 18 (2005)

17. Nayar, S., Murase, -H., Nene, S.: Columbia object image library: Coil (1996)

18. Bunke, H., Neuhaus, M.: Edit distance based kernel functions for structural pattern classification. Pattern Recognition 39(10), 1852-1863 (2006)

19. Hancock, E.R., Qiu, H.: Clustering \& embedding using commute times. IEEE Transactions on Pattern Analysis \& Machine Intelligence(PAMI) 29(11), 1873-1890 (2007)

20. Smola, A., Muller, -K.R., Scholkopf, B.: Nonlinear component analysis as a kernel eigenvalue problem. Neural Computation 10, 1299-1319 (1998)

21. Smola, A.J., Muller, -K.-R., Scholkopf, B.: Kernel principal component analysis. In: Scholkopf, B., Burges, C.J.C., Smola, A.J. (eds.) Advances in Kernels MethodsSupport Vector Learning, pp. 327-352 (1999)

22. Christianini, N., Shawe-Taylor, J.: Kernel Methods for Pattern Analysis. Cambridge University Press, New York (2004)

23. Borgwardt, K.M., Schraudolph, -N.N., Vishwanathan, S.: Fast computation of graph kernels. In: Adv. NIPS (2007)

24. Smola, -J., Vishwanathan, S.V.N.: Fast kernels for strings and tree matching. Advances in Neural Information Processing Systems 15 (2003) 